\title{
Aprendizado de métrica de distância sobre o desempenho de um classificador Máquina de Vetor de Suporte $(S V M)$.
}

\author{
Leonardo Vieira da Costa* Cristiano Leite de Castro** \\ * Programa de Pós-Graduação em Engenharia Elétrica, Universidade \\ Federal de Minas Gerais Brasil (Tel: 31-99619-4092; e-mail: \\ lvieirad@ufmg.br). \\ ** Departamento de Engenharia Elétrica, Universidade Federal de \\ Minas Gerais Brasil (e-mail: crislcastro@ufmg.br)
}

\begin{abstract}
The Metric Learning has been of great use in the development of algorithms machine learning, standing out for the solution of problems related to the performance of classifiers. An efficient classification tool is the Support Vector Machine $(S V M)$, designed to achieve good generalization capability. This study aims to propose the use of distance metric learning on the performance of an $S V M$ classifier. This method uses the Mean Square Error (MSE) to find the best Kernel in the training base, and thus apply it to the test base. In the realized experiments, it was verified that the adjustment of the Kernel parameters through a metric learning approach can considerably improve the performance of an $S V M$ classifier.

Resumo: O aprendizado de métrica tem sido de grande utilidade no desenvolvimento de algoritmos de aprendizado de máquinas, destacando-se para a solução de problemas relacionados com o desempenho de classificadores. Uma ferramenta de classificação eficiente é a Máquina de Vetor de Suporte $(S V M)$, projetada para obter uma boa capacidade de generalização. Este trabalho tem como objetivo propor a utilização de um aprendizado de métrica de distância sobre o desempenho de um classificador $S V M$. Tal método utiliza-se do Erro Quadrático Médio (MSE) para encontrar o melhor Kernel na base de treinamento, e assim aplicá-lo à base de teste. Nos experimentos realizados, foi constatado que o ajuste dos parâmetros do Kernel por meio de uma abordagem de aprendizagem métrica pode melhorar consideravelmente o desempenho de um classificador $S V M$.
\end{abstract}

Keywords: Metric Learning; Machine Learning; Support Vector Machine; Kernel; Mean Square Error.

Palavras-chaves: Aprendizado de Métrica; Máquina de Vetor de Suporte; Kernel; Erro Quadrático Médio.

\section{INTRODUÇÃO}

O bom desempenho de um algoritmo de aprendizado de máquinas depende do tipo de métrica utilizada para a avaliação da distância ou, do lado avesso, da medida de semelhança entre pontos ou vetores no espaço de entrada. No algoritmo de qualquer classificador, dependendo do cálculo das distâncias entre vetores, podem-se obter diferentes tipos de classificação para novos dados ainda não vistos. Neste sentido, o objetivo do aprendizado de uma métrica consiste em proporcionar um reescalonamento dos dados de modo que a solução encontrada melhore a acurácia de um determinado classificador.

É importante ressaltar o trabalho do Xing et al. (2003) que tem como objetivo o aprendizado de métrica de distância como um problema de programação convexa restrita. Tal trabalho utiliza uma métrica de distância global com o objetivo de minimizar a distância entre os pares de dados. De outra forma, o autor Lebanon (2003) utiliza o aprendizado a partir de exemplos rotulados e em função da distância de uma geometria da informação. Já para autor Bar-Hillel et al. (2003) a métrica de distância tem como objetivos minimizar a distância entre os pontos de dados dentro dos mesmos rótulos e maximizar a distância entre os pontos de dados de rótulos diferentes. Wang et al. (2014) propôs um framework para sugerir novos métodos de aprendizado de métrica, que pode ser implementado utilizando máquina de vetores de suporte (SVM).

Um classificador muito utilizado é Máquina de Vetor de Suporte $(S V M)$ desenvolvida por Boser et al. (1992). A $S V M$ constrói um hiperplano ou conjunto de hiperplanos em um espaço de alta dimensão, que pode ser usado para classificação, regressão ou outras tarefas. Uma boa separação é obtida pelo hiperplano que tem a maior distância até o ponto de dados de treinamento mais próximo de qualquer classe, chamada como margem funcional. Em geral, quanto maior a margem, menor o erro de generalização do classificador com base na teoria de aprendizado estatístico. A sua fórmula resulta em um problema convexo que pode 
ser resolvido por programação quadrática demonstrada em Vapnik (1999) conforme equação 1.

$$
\begin{array}{r}
\max _{\alpha} f(\alpha)=\sum_{i=1}^{n} \alpha_{i}-\frac{1}{2} \sum_{i, j=1}^{n} \alpha_{i} \alpha_{j} y_{i} y_{j} k\left(\overrightarrow{x_{i}}, \overrightarrow{x_{j}}\right) \\
\text { sujeito a } 0 \leq \alpha_{i} \leq C e \sum_{i=1}^{n} \alpha_{i} y_{i}=0 \\
\text { onde } \mathrm{k}\left(\overrightarrow{x_{i}}, \overrightarrow{x_{j}}\right)=\exp \left(-\frac{\left\|\overrightarrow{x_{i}}-\overrightarrow{x_{j}}\right\|^{2}}{2 \sigma^{2}}\right)
\end{array}
$$

Onde são os coeficientes de Lagrange, $C$ é a variável de folga e $K$ é a função de Kernel. A escolha tanto do Kernel e dos parâmetros influenciam diretamente na qualidade do classificador demostrado em Scholkopf and Smola (2004).

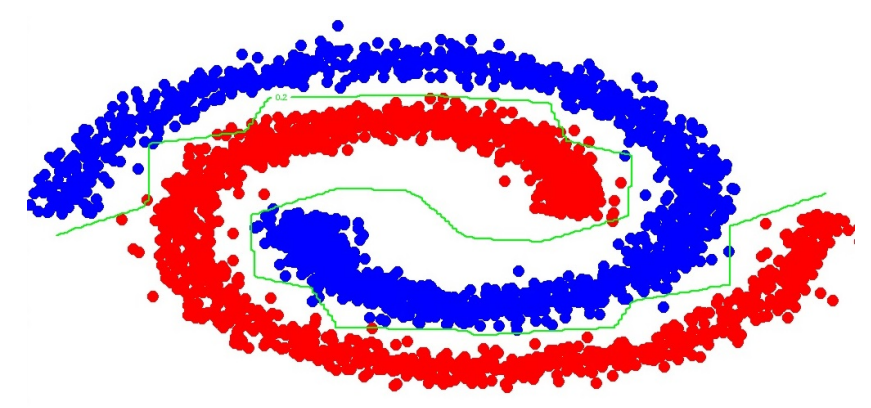

Figura 1. $\sigma=0.02$ sobre-ajuste.

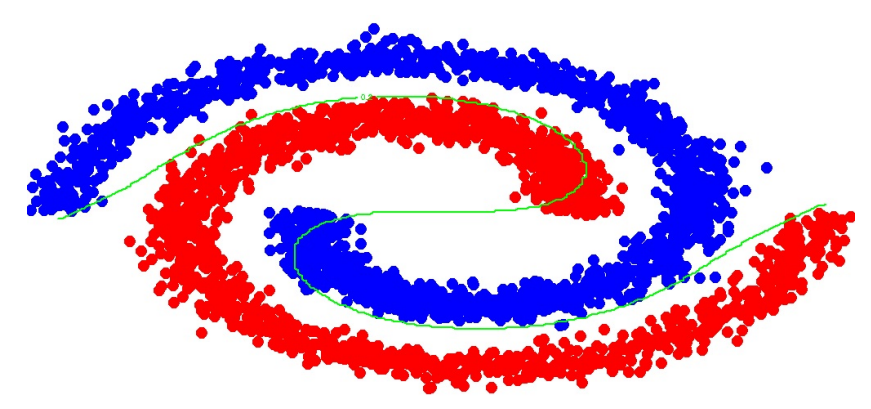

Figura 2. $\sigma=0.3$ sub-ajuste.

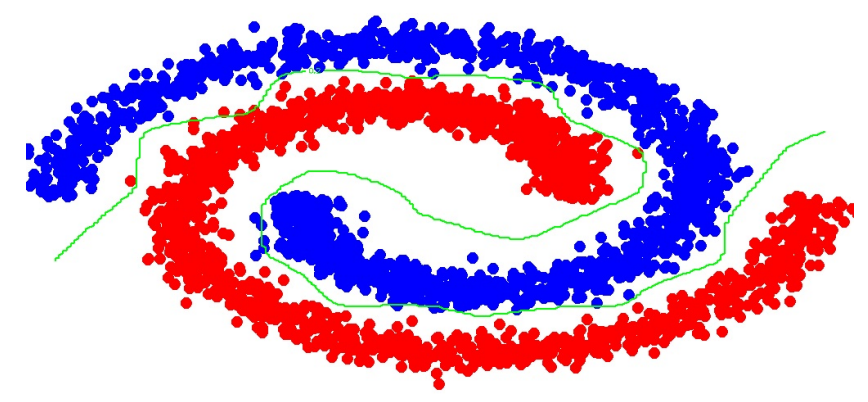

Figura 3. $\sigma=$ Distância média dos $N$ vizinhos.

A necessidade de escolher um bom valor para parâmetro $\sigma$ conforme Zaidi and Squire (2010) é de extrema importância para o desempenho do classificador. Valores muito altos podem gerar um sub-ajuste, em contrapartida, valores muito baixos geram sobre-ajuste. Nas Fig. 1, 2 e 3 verificase o efeito da variação do parâmetro $\sigma$ na classificação dos dados.

\section{METODOLOGIA}

Nesta seção, é descrito o algoritmo de aprendizado de métrica de distância sobre o desempenho de um classificador $S V M$. A proposta desse trabalho é encontrar uma métrica ideal minimizando diretamente o erro quadrático médio na base de treinamento. Tal minimização irá definir o melhor Kernel, para posteriormente aplicá-lo na base de teste.

\subsection{Erro quadrático Médio (MSE)}

Para avaliar o desempenho de um método de aprendizagem estatística de um conjunto de dados, é preciso alguma metodologia para medir o quão próximas às previsões estarão dos dados observados. Ou seja, é necessário quantificar a extensão para o qual o valor de resposta previsto para uma determinada observação está próximo do verdadeiro valor de resposta para essa observação. No ajuste de regressão, a medida mais comumente usada é o erro quadrático médio (MSE), dado pela equação 2.

$$
M S E=\frac{1}{n} \sum_{i=1}^{n}\left(y_{i}-\hat{f}\left(x_{i}\right)\right)^{2}
$$

Onde $\hat{f}_{\left(x_{i}\right)}$ é a predição que $\hat{f}$ dá para a $i$-enésima observação James et al. (2014). O MSE será pequeno se as respostas previstas estiverem muito próximas das respostas verdadeiras, e será grande se as observações forem substancialmente diferentes entre o previsto e o verdadeiro.

\subsection{Distância Minkowski}

A proximidade entre dois dados $x_{i}$ e $x_{j}$ é denotada por $d\left(x_{i}, x_{j}\right)$. Uma maneira de medir a similaridade entre os atributos contínuos é através do cálculo de distância entre eles. Para que a distância seja considerada uma métrica deve-se satisfazer as seguintes condições:

- $d\left(x_{i}, x_{j}\right) \geq 0$

- $d\left(x_{i}, x_{j}\right)=0$,se e somente se $x_{i}=x_{j}$;

- $d\left(x_{i}, x_{j}\right)=d\left(x_{j}, x_{i}\right)$

- $d\left(x_{i}, x_{l}\right) \leq d\left(x_{i}, x_{j}\right)+d\left(x_{j}, x_{l}\right)$.

Uma das distâncias utilizadas no cálculo de similaridade entre dois dados é à distância de Minkowski. Tal distância é calculada pela equação 3 onde $d$ é o número de atributos do dado conforme Jain et al. (1988).

$$
d\left(x_{i}, x_{j}\right)=\sqrt[p]{\sum_{k=1}^{d}\left(\left|x_{i k}-x_{j k}\right|\right)}, p \geq 1
$$

Quando $p=1$ à distância Minkowski se equivale à distância Manhattan, da mesma forma quando $p=2$ à distância Minkowski se equivale à distância euclidiana.

\subsection{Função de Kernel SVM}

Máquinas de Vetores de Suporte $(S V M)$ são classificadores que estimam o resultado $y_{j}$ de um valor qualquer de $x_{j}$ através de uma soma ponderada de resultados $y_{i}$ pelo valor da função de Kernel $K\left(x_{j} ; x_{i}\right)$ demonstrado por Yang and 
Jin (2006). A função de Kernel proposta neste trabalho é uma métrica mais geral da equação (3) está descrita na equação 4 .

$$
d_{A}^{2}\left(x_{i}, x_{j}\right)=\left\|x_{i}-x_{j}\right\|_{A}^{2}=\left(x_{i}-x_{j}\right)^{T} A\left(x_{i}-x_{j}\right)
$$

Onde $A$ é uma matriz de distância Minkowski calculada pela equação 4 variando o valor de $p$.

\subsection{Toda a Metodologia}

Dado um determinado problema, como por exemplo, o de classificação, faz-se necessária a realização de diversas etapas a fim de implementar o método:

- Primeiramente calcula-se os Kernels do $S V M$ variando a matriz $A$ de distância Minkowski em função do $p$.

- Posteriormente calcula-se o menor erro (MSE) quadrático médio nos dados de treinamento.

- Após realizado o ranqueamento dos Kernels em função do MSE, escolhe-se o Kernel com menor MSE e aplica-se aos dados de teste.

- Por fim verifica-se a acurácia do modelo proposto

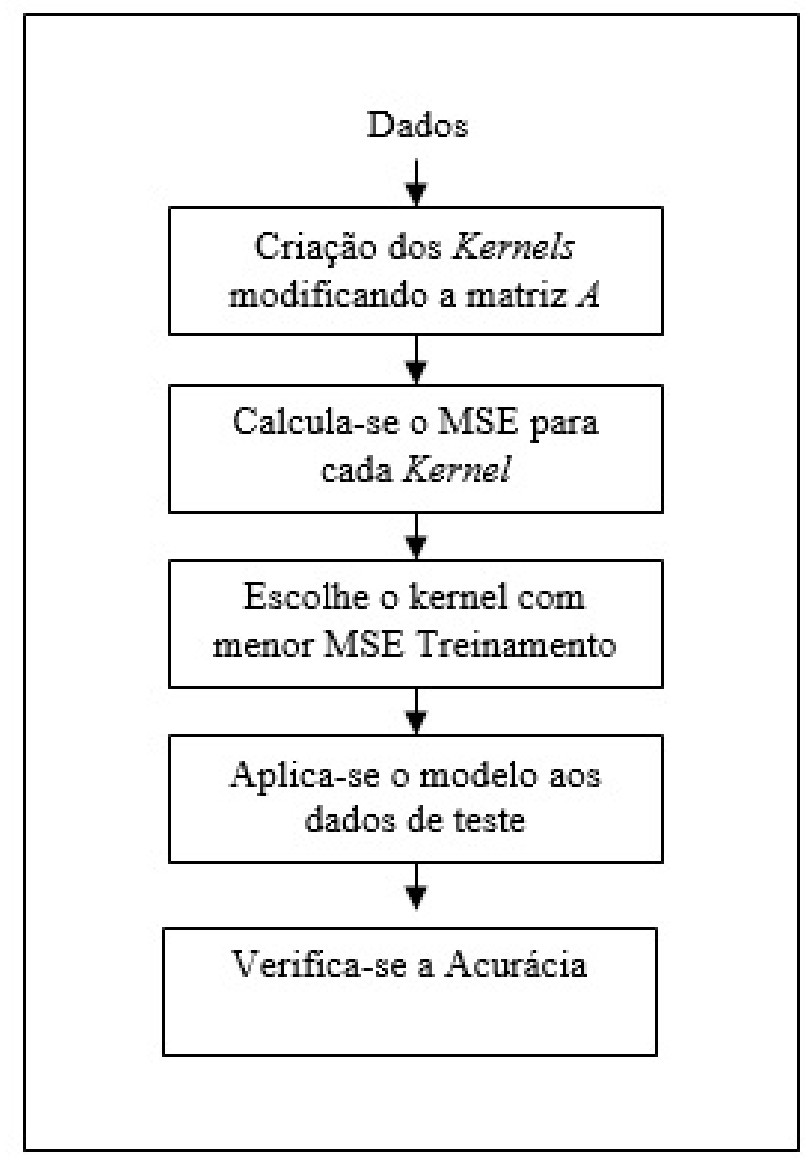

Figura 4. Estrutura do método proposto.

A Fig. 4 ilustra os principais componentes de todo o algoritmo do método proposto e o modo como eles se conectam. Desta forma, a metodologia desse trabalho mostra que o aprendizado de métrica acontece na variação do valor do parâmetro $p$ da métrica Minkowoski. Tal parâmetro tem um valor específico dependendo da base de treino, correspondendo assim a uma métrica diferente aprendida após realizado o ranqueamento dos Kernels em função do $M S E$. Posteriormente ao ranqueamento, escolhese o Kernel com menor MSE e aplica-se aos dados de teste.

\section{RESULTADOS E DISCUSSÃO}

A fim de testar o método proposto para o problema de classificação, cinco bases de dados encontradas em Dua and Graff (2007) foram escolhidas neste trabalho. As bases de dados serão descritas para melhor exemplificar o desempenho do método proposto frente a Máquinas de Vetores de Suporte (SVM) com Kernel Gaussiano. Toda a implementação foi realizada utilizando o software $R$ Team (2013). Utilizou-se também o pacote 'kernlab' que disponibiliza o algoritmo $S V M$.

\subsection{Base de dados Default}

A base de dados Default é um conjunto de registros dos clientes para uma empresa de cartão de crédito. Ela possui 4 características organizadas em 10.000 amostras e apresentado na Tabela 1 .

A variável escolhida como resposta foi a Student. Apesar do grande número de amostras a base de dados apresenta uma distribuição bem definida. Por esse motivo os dois métodos obtiveram ótimos desempenhos na base de teste.

Tabela 1. Informações sobre a base de dados Default.

\begin{tabular}{cc} 
Atributo & Descrição \\
\hline Default & Yes ou No \\
Student & Yes ou No \\
Income & Contínuo entre 0 e 2654.323 \\
Balance & Contínuo entre 771.9677 e 73554.23 \\
\hline
\end{tabular}

\subsection{Base de dados Iris}

A base de dados Iris tem como objetivo classificar a espécie de planta Iris. O atributo Species possui três classes: Iris Setosa, Iris Versicolour, Iris Virginica. A Base é composta por 5 características e 150 amostras apresentado na Tabela 2 .

Tabela 2. Informações sobre o Bando de Dados Iris.

\begin{tabular}{cc} 
Atributo & Descrição \\
\hline Species & Setosa, Versicolour e Virginica \\
sepal length & Contínuo entre 4.3 e 7.9 \\
sepal width & Contínuo entre 2 e 4.4 \\
petal length & Contínuo entre 1 e 6.9 \\
spetal width & Contínuo entre 0.1 e 2.5 \\
\hline
\end{tabular}

Devido à simplicidade da base e a clara diferenciação entre as classes conforme Fig 5, o método proposto e o $S V M$ foram capazes de classificar corretamente quase todas as amostras na base de teste.

\subsection{Base de dados 1985 Auto Imports}

O conjunto de dados 1985 Auto Imports consiste de 193 amostras. Para cada amostra, 26 atributos podem ser observados como descrito na Tabela 3. 


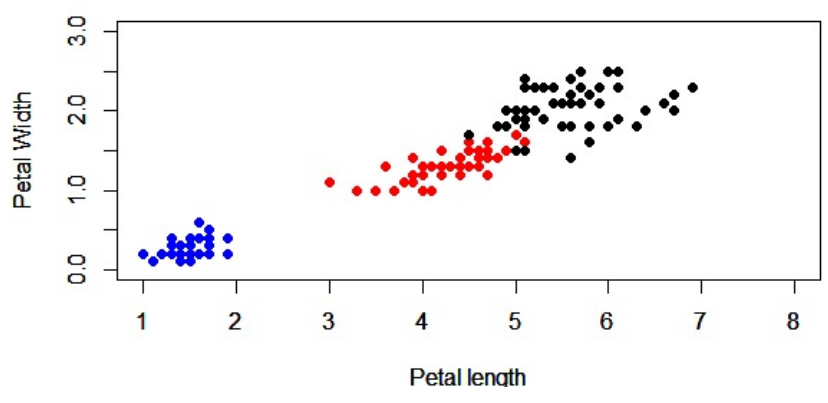

Figura 5. Distribuição dos dados da base de dados Iris.

Tabela 3. Informações sobre a base de dados 1985 Auto Imports.

\begin{tabular}{|c|c|}
\hline Atributo & Descrição \\
\hline symboling & $-3,-2,-1,0,1,2,3$ \\
\hline normalized-losses & Contínuo entre 65 e 256. \\
\hline fuel-type & diesel, gas. \\
\hline aspiration & std, turbo \\
\hline num-of-doors & four, two \\
\hline body-style & hardtop, wagon, sedan, hatchback \\
\hline drive-wheels & 4wd, fwd, rwd. \\
\hline engine-location: & front, rear \\
\hline wheel-base & Contínuo entre 86.6 e 120.9 . \\
\hline length & Contínuo entre 141.1 e 208.1 \\
\hline width & Contínuo entre 60.3 e 72.3 \\
\hline height & Contínuo entre 47.8 e 59.8 \\
\hline curb-weight & Contínuo entre 1488 e 4066 \\
\hline engine-type & dohc, dohcv, l, ohc, ohcf, ohcv, rotor \\
\hline num-of-cylinders & eight, five, four, six, three, twelve, two \\
\hline engine-size & Contínuo entre 61 e 326 \\
\hline fuel-system & 1bbl, 2bbl, 4bbl, idi, mfi, mpfi, spdi, spfi \\
\hline bore & Contínuo entre 2.54 e 3.94 \\
\hline stroke & Contínuo entre 2.07 e 4.17 \\
\hline compression-ratio & Contínuo entre 7 e 23 \\
\hline horsepower & Contínuo entre 48 e 288 \\
\hline peak-rpm & Contínuo entre 4150 e 6600 \\
\hline city-mpg & Contínuo entre 13 e 49 \\
\hline highway-mpg & Contínuo entre 16 e 54 \\
\hline price & Contínuo entre 5118 e 45400 \\
\hline
\end{tabular}

Uma nova característica foi criada para definir a classificação das amostras baseada na característica existente symboling. Tal característica foi dividida em duas classes, a classe de clientes com baixo risco de análise do seguro que tem valores de symboling menores ou iguais a zero, e a classe com alto risco de análise do seguro que tem valores de symboling maiores do que zero. O método proposto e o $S V M$ foram capazes de classificar corretamente quase todas as amostras na base de teste.

\subsection{Base de dados Scale Weight \& Distance}

A base de dados Scale Weight \& Distance tem como objetivo modelar resultados experimentais psicológicos. O atributo Class Name possui três classes: left, right $e$ balanced. A base é composta por 5 características e 625 amostras apresentado em Tabela 4.

O método proposto e o $S V M$ apresentaram uma acurácia muito semelhante na base de teste
Tabela 4. Informações sobre a base de Dados Scale Weight \& Distance

\begin{tabular}{cc} 
Atributo & \multicolumn{1}{c}{ Descrição } \\
\cline { 2 - 3 } Class Name & left, right e balanced \\
Left-Weight & Contínuo entre 1 e 5 \\
Left-Distance & Contínuo entre 1 e 5 \\
Right-Weight & Contínuo entre 1 e 5 \\
Right-Distance & Contínuo entre 1 e 5 \\
\hline
\end{tabular}

\subsection{Base de dados House Boston}

A base de dados House Boston tem como objetivo mostrar os valores das casas do subúrbio de Boston. Ele possui 13 características de valores contínuos com 5066 amostras apresentado em Tabela 5.

Tabela 5. Informações sobre a base de dados House Boston.

\begin{tabular}{|c|c|}
\hline Atributo & Descrição \\
\hline CRIM & $\begin{array}{l}\text { Taxa de criminalidade per capita por ci- } \\
\text { dade }\end{array}$ \\
\hline ZN & $\begin{array}{l}\text { Proporção de terrenos residenciais por zo- } \\
\text { nas para lotes } 25.000 \text { sq.ft. }\end{array}$ \\
\hline INDUS & $\begin{array}{l}\text { Proporção de negócios não-varejo por ci- } \\
\text { dade }\end{array}$ \\
\hline CHAS & Lado da cidade em relação ao Rio Charles \\
\hline NOX & $\begin{array}{l}\text { Concentração de óxidos nítricos (partes } \\
\text { por } 10 \text { milhões) }\end{array}$ \\
\hline $\mathrm{RM}$ & Número médio de quartos por habitação \\
\hline AGE & $\begin{array}{l}\text { Proporção de IDADE das unidades ocupa- } \\
\text { das pelo proprietário construídas antes de } \\
1940\end{array}$ \\
\hline DIS & $\begin{array}{l}\text { Distâncias ponderadas a cinco centros de } \\
\text { emprego de Boston }\end{array}$ \\
\hline RAD & $\begin{array}{l}\text { Índice RAD de acessibilidade a estradas } \\
\text { principais }\end{array}$ \\
\hline TAX & $\begin{array}{l}\text { Imposto sobre o Valor Agregado por } \$ \\
10.000\end{array}$ \\
\hline PTRATIO & Relação aluno-professor por cidade \\
\hline B & $\begin{array}{l}1000(\mathrm{Bk}-0,63){ }^{\wedge} 2 \text { onde } \mathrm{Bk} \text { é a proporção } \\
\text { de negros por cidade }\end{array}$ \\
\hline LSTAT & $\%$ status da população com menor renda \\
\hline MEDV & $\begin{array}{l}\text { Valor médio das casas ocupadas por pro- } \\
\text { prietários em } \$ 1000\end{array}$ \\
\hline
\end{tabular}

Como o objetivo deste trabalho é calcular a acurácia do modelo proposto, foi criada uma nova característica que leva em conta o valor médio das casas ocupadas por proprietários em $\$ 1000$ (MEDV). Considerou-se casas com valores menores ou iguais a 21.8 como casas da classe "inferiores", e casas acima dos valores de 21.8 como casas da classe "superiores". Depois dessa operação a base ficou balanceada entre as duas classes e posteriormente foram aplicados os dois métodos para verificar a acurácia. O método proposto teve um desempenho muito superior na base de teste

\subsection{Comparação dos métodos nas cinco bases de dados}

A Fig. 5 mostra a comparação do método proposto neste artigo com o $S V M$ nas cinco bases de dados. Os dados foram divididos entre treinamento e teste, e posteriormente computou-se o percentual da acurácia de cada método na base de teste.

O método proposto possui uma acurácia muito semelhante à do método $S V M$ em quatro bancos de dados, utilizando 


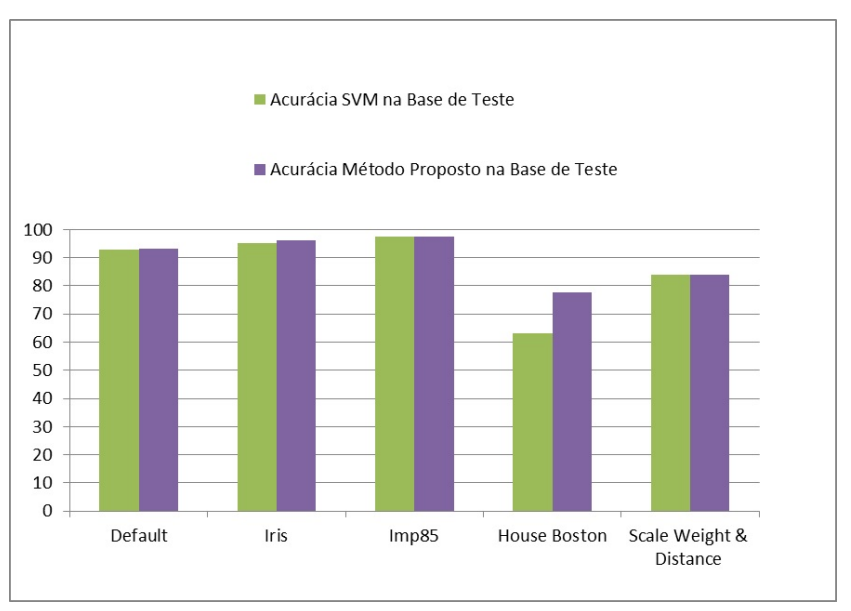

Figura 6. Gráfico com a acurácia dos modelos em \%.

a base teste. O ponto de destaque foi a superioridade da acurácia do método apresentado utilizando-se a base de teste do banco de dados do House Boston, o que indica uma boa capacidade de generalização do modelo criado.

\section{CONCLUSÃO}

Este artigo propõe a utilização de um aprendizado de métrica de distância sobre o desempenho de um classificador $S V M$. O método proposto utiliza-se do erro quadrático médio (MSE) para ranquear o melhor Kernel na base de treinamento. Uma vez definido o Kernel que possui o menor MSE aplica-se o mesmo na base de teste e verifica-se a acurácia.

Os resultados alcançados nos experimentos realizados em cinco bases de dados, o modelo proposto demostra que na base de dados House Boston, a acurácia no conjunto de teste foi melhor do que o $S V M$. Foi descoberto que o ajuste dos parâmetros do Kernel por meio de uma abordagem de aprendizagem métrica pode melhorar o desempenho de um classificador $S V M$.

Em trabalhos futuros serão investigadas variações na modificação da matriz de distância para encontrar o melhor Kernel.

\section{REFERÊNCIAS}

Bar-Hillel, Aharon, Hertza, Tomer, Shental, Noam, Weinshall, and Daphna (2003). Learning distance functions using equivalence relations. Proceedings of the 20th International Conference on Machine Learning (ICML03), 11-18.

Boser, B., Guyon, I., and Vapnik, V. (1992). A training algorithm for optimal margin classifiers. Proceedings of the Fifth Annual Workshop on Computational Learning Theory, ACM Press, pp. 144-152.

Dua, D. and Graff, C. (2007). Machine learning repository - uci. University of California, Irvine, School of Information and Computer Sciences, URL http://archive.ics.uci.edu/ml.

Jain, A., and Dubes, R. (1988). Algorithms for clustering data. Prentice-Hall.

James, G., Witten, D., Hastie, T., and Tibshirani, R. (2014). An introduction to statistical learning: with applications in r. Springer Publishing Company, Incorporated.

Lebanon, G. (2003). Flexible metric nearest neighbor classification. Proc. Uncertainty in Artificial Intelligence.

Scholkopf, B. and Smola, A. (2004). Learning with kernels, support vector machines, regularization, optimization and beyond. The MIT Press.

Team, R.C. (2013). R: A language and environment for statistical computing. $R$ Foundation for Statistical Computing, Vienna, Austria, URL http://www.Rproject.org/.

Vapnik, V. (1999). An overview of statistical learning theory. IEEE Transactions on Neural Networks 10, no. 5, 988-999.

Wang, Faqiang, Zuo, Wangmeng, Zhang, Lei, Meng, Deyu, Zhang, and David (2014). A kernel classification framework for metric learning. IEEE transactions on neural networks and learning systems, 26(9), 1950-1962.

Xing, P, E., Jordan, I, M., Russell, J, S., Ng, and Y, A. (2003). Distance metric learning with application to clustering with side-information. Advances in neural information processing systems, 521-528.

Yang, L. and Jin, R. (2006). Distance metric learning: A comprehensive survey. Department of Computer Science and Engineering Michigan State University.

Zaidi, N. and Squire, D. (2010). Svms and data dependent distance metric. Clayton School of Information Technology, Monash University, Clayton, VIC, 3800. 